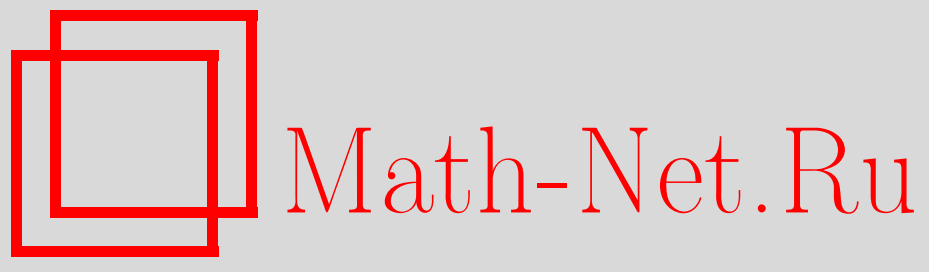

Р. Дж. Муллани, Двойные пространства Фано с большим сингулярным локусом, Матем. заметки, 2010, том 87, выпуск 3, 472-476

DOI: https://doi.org/10.4213/mzm8679

Использование Общероссийского математического портала Math-Net.Ru подразумевает, что вы прочитали и согласны с пользовательским соглашением http://www .mathnet.ru/rus/agreement

Параметры загрузки:

IP : 54.80 .97 .219

26 апреля 2023 г., 10:47:26

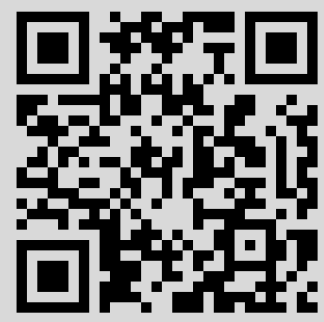




\section{Двойные пространства Фано с большим сингулярным локусом}

\section{Р. Дж. Муллани}

1. Формулировка основного результата. Всюду в дальнейшем предполагается, что $m \geqslant 6$ - целое фиксированное число. Выберем линейное подпространство $P \subset \mathbb{P}^{m}$ коразмерности $k$ такое, что выполняется неравенство $m \leqslant k(k-3) / 2+2$. Пусть $W=$ $W_{2 m} \subset \mathbb{P}^{m}$ - порождающая гиперповерхность, сингулярная в каждой точке линейного подпространства $P$ (такая, что $P=\operatorname{Sing} W$ - геометрическое место (локус) двойных точек порождающей гиперповерхности $W$ ). Ясно, что если выбрать уравнения $x_{0}=$ $\cdots=x_{k-1}=0$ для $P$ и координаты $x_{k}, \ldots x_{m}$ на плоскости, то $W$ задается уравнением

$$
f\left(x_{*}\right)=\sum_{i, j=0}^{k-1} x_{i} x_{j} h_{i j}\left(x_{*}\right)=0,
$$

где $h_{i j}\left(x_{*}\right)=h_{j i}\left(x_{*}\right)$ - однородные полиномы степени $2 m-2$ относительно $x_{0}, \ldots, x_{m}$. Пусть $\pi: V \rightarrow \mathbb{P}^{m} \supset W_{2 m}$ - двойное пространство индекса 1 , разветвленное над $W$, которое можно определить явным образом как гиперповерхность, задаваемую уравнением

$$
x_{m+1}^{2}=f\left(x_{0}, \ldots, x_{m}\right)
$$

в весовом проективном пространстве $\mathbb{P}^{m+1}(1, \ldots, 1, m)$.

Многообразие $V$ является факторным $m$-мерным многообразием с сингулярным локусом $P^{*}=\pi^{-1}(P)$. Кроме того, $V$ - многообразие Фано индекса 1 , т.е. Pic $V=\mathbb{Z} K_{V}$, где $K_{V}=-\pi^{*} H$ и $H-$ класс гиперплоскости в $\mathbb{P}^{m}$. Эти свойства многообразия $V$ следуют из того, что двойное накрытие $\pi: V \rightarrow \mathbb{P}^{m}$ может быть продолжено в двойное накрытие $\pi^{+}: V^{+} \rightarrow \widetilde{\mathbb{P}}^{m}$, где $\varphi: V^{+} \rightarrow V$ и $\widetilde{\varphi}: \widetilde{\mathbb{P}}^{m} \rightarrow \mathbb{P}^{m}-$ раздутия подпространств $P^{*}$ и $P$. Так как $W$ является порождающей гиперповерхностью, ее строгое преобразование $\widetilde{W} \subset \widetilde{\mathbb{P}}^{m}$ гладкое и, таким образом, $V^{+}$тоже гладкое. Теперь основной результат этой работы можно сформулировать следующим образом.

Теорема. Многообразие $V$ бирачионалъно супержесткое.

СлеДСтвиЕ. (i) Невозможно расслоить многообразие $V$ на равнолинейчатые многообразия с помощью некоторого нетривиального рационального отображения.

(ii) Многообразие $V$ нерационально.

(iii) Группь бирациональных и бирегулярных отображений в себл совпадают: $\operatorname{Bir} V=$ Aut $V=\mathbb{Z} / 2 \mathbb{Z}$.

Теория бирациональной жесткости восходит к классической работе [1] Исковского и Манина о трехмерных квадриках. Основные определения этой теории даны в работе [2]. Из вышеприведенной теоремы следует, что для любой подвижной линейной системы $\Sigma$ на $V$ ее виртуальный порог канонического присоединения $c_{\text {virt }}(\Sigma, V)$ равен порогу $c(\Sigma, V)$ на $V$. В силу этого равенства утверждения следствия доказываются стандартным образом [2].

Перечислим существующие результаты, тесно связанные с тематикой данной работы.

Пример. (i) При $m \geqslant 3$ гладкое двойное пространство $\pi: V \rightarrow \mathbb{P}^{m} \supset W_{2 m}$ индекса 1 , разветвленное над гладкой гиперповерхностью $W$ степени $2 m$, является бирационально супержестким (см. [3]).

(ii) Предположения пункта (i) сохраняются, но $W$ имеет единственную особенность в точке $x \in W, \operatorname{mult}_{x} W=2 l, l \leqslant m-2$, а касательный конус $T_{x} W$ определяет гладкую 
гиперповерхность степени $2 l$ в проективном пространстве $\mathbb{P}\left(T_{x} \mathbb{P}^{m}\right) \cong \mathbb{P}^{m-1}$. Тогда многообразие $V$ бирационально супержесткое (см. [4]). Для случая $m=3$ предполагается, что $W$ максимально порождающее (более подробное изложение дано в [4; предложение 1]).

(iii) Предположения пункта (i) сохраняются, но $W$ имеет кратные изолированные особенности, каждая из которых удовлетворяет условиям пункта (ii). Тогда $V$ бирационально супержесткое (см. [5]).

(iv) Бирациональная геометрия трехкратных двойных пространств с двойной линией оказывается более сложной, поскольку эти пространства могут расслаиваться на поверхности дель Пеццо (см. [6]).

2. План и начало доказательства. Пусть $V$ - не супержесткое многообразие. По определению это значит, что можно зафиксировать некоторую подвижную линейную систему $\Sigma \subset\left|-n K_{V}\right|$ такую, что

$$
c_{\mathrm{virt}}(\Sigma)<c(\Sigma, V)=n .
$$

Из стандартных рассуждений теории бирациональной жесткости [2] следует, что линейная система $\Sigma$ имеет максимальную сингулярность, т.е. для некоторого неприводимого исключительного дивизора $E^{\sharp} \subset V^{\sharp}$ на некоторой модели $\psi: V^{\sharp} \rightarrow V$ многообразия $V$ выполняется неравенство Нётер-Фано:

$$
\operatorname{ord}_{E^{\sharp}} \psi^{*}(\Sigma)>n a\left(E^{\sharp}\right) .
$$

Иначе говоря, пара $(V, \Sigma / n)$ не является канонической, а $E^{\sharp}$ - некоторая неканоническая особенность этой пары. Пусть $B=\psi\left(E^{\sharp}\right)$ - ее центр на $V$. Теперь, рассуждая как в работе [2], исключим возможность того, что $B \not \subset P^{*}=\operatorname{Sing} V:$ если $\operatorname{codim} B=2$, то можно дословно повторить доказательство, приведенное в [2; гл. 2, предложение 2.9], так как $\operatorname{codim} P^{*} \geqslant 5$. Иначе говоря, $\operatorname{codim} B \geqslant 3$, и проводя вычисления для точки, порождающей $B$, которая не является сингулярной на $V$, получим неравенство

$$
\operatorname{mult}_{B} Z>4 n^{2}
$$

для самопересечения $Z=\left(D_{1} \circ D_{2}\right)$ линейной системы $\Sigma$ (подробное изложение дано в [2; гл. 2, теорема 2.2]). Неравенство (1) невозможно, так как $\operatorname{deg} Z=2 n^{2}$. Следовательно, можно считать, что $B \subset \operatorname{sing} V=P^{*}$.

3. Максимальные особенности над подмногообразием в сингулярном локусе. Выше было показано, что так как $W$ - порождающая гиперповерхность, раздутие $\varphi: V^{+} \rightarrow V$ сингулярного локуса $P^{*}$ является гладким многообразием с исключительным дивизором $E^{+}=\varphi^{-1}\left(P^{*}\right)$. Подмногообразием $E^{+}$гладкое, и $\varphi_{E^{+}}: E^{+} \rightarrow P^{*} \cong P-$ его расслоение на квадрики. В обозначениях, принятых выше,

$$
h_{i j}\left(x_{0}, \ldots, x_{m}\right)=P_{i j}\left(x_{k}, \ldots, x_{m}\right)+h_{i j}^{+},
$$

где каждый член однородных многочленов $h_{i j}^{+}$содержит по крайней мере одну из переменных $x_{0}, \ldots, x_{k-1}$. В этих обозначениях, можно идентифицировать слой расслоения $\varphi_{E^{+}}$над точкой $\left(0: \cdots: 0: a_{k}: \cdots: a_{m}\right)$ как квадратичную гиперповерхность

$$
x_{m+1}^{2}=\sum_{i, j=0}^{k-1} x_{i} x_{j} P_{i j}\left(a_{k}, \ldots, a_{m}\right) .
$$

В нашем дальнейшем доказательстве основные технические трудности возникают из-за того, что некоторые слои в $E^{+} / P^{*}$ могут быть сингулярными. Поэтому, чтобы закончить доказательство, мы должны в точности понять, до какой степени слои квадрики могут вырождаться. 
Заметим, что если $B=P^{*}$ является полным сингулярным локусом, то можно использовать рассуждения, почти идентичные рассуждениям в гладком случае: изменяя доказательство, приведенное в работе [2], придем к неравенству mult $_{B} Z>4 n^{2}$ для самопересечения $Z=\left(D_{1} \circ D_{2}\right)$ линейной системы $\Sigma$, а затем придем к противоречию, как и в [2].

$\mathrm{K}$ сожалению, этот подход нельзя применить и в случае произвольного подмногообразия $B \subset P^{*}$. Дело в том, что, раздувая $B$, а затем центр максимальной особенности на раздутии, и так далее, мы получим последовательность сингулярных многообразий, особенности которых трудно исследовать. Поэтому, нужен другой подход.

Во-первых, пусть $\Pi \subset \mathbb{P}^{m}$ - порождающее линейное подпространство размерности $\operatorname{codim} B \geqslant 6, V_{\Pi}=\pi^{-1}(\Pi)$ - соответствующее двойное накрытие, и $\Sigma_{\Pi}=\left.\Sigma\right|_{V_{\Pi}}-$ подвижная линейная система, определенная как сужение линейной системы $\Sigma$ на $V_{\Pi \text { п }}$ Так как П - порождающая гиперповерхность, то пара $\left(V_{\Pi}, \Sigma_{\Pi} / n\right)$ является канонической, т.е. $\Sigma_{\Pi}$ имеет максимальную особенность, центр которой является точкой сингулярного локуса $P^{*} \cap V_{\Pi}$; именно, одной из точек пересечения $B \cap V_{\Pi}$. Следовательно, задача свелась к случаю, в котором центр максимальной особенности оказывается точкой сингулярного локуса. Чтобы изучить этот случай, нам надо исследовать вырожденные слои многообразия $E^{+}$над $P^{*} \cong P$.

4. Вырождающиеся квадрики. Пусть $S$ - пространство симметричных $k \times k$-матриц с $\mathbb{C}$-элементами, это пространство имеет размерность $k(k+1) / 2$, и пусть $\mathbf{S}$ - пространство симметричных $k \times k$-матриц, элементы которых являются однородными полиномами степени $2 m-2$ относительно $x_{k}, \ldots, x_{m}$. Так как число мономов степени $n$ относительно $r$ переменных равно $\left(\begin{array}{c}n+r-1 \\ n\end{array}\right)$, то пространство $\mathbf{S}$ имеет размерность

$$
\frac{k(k+1)}{2}\left(\begin{array}{c}
3 m-k-2 \\
2 m-2
\end{array}\right) .
$$

Пусть $(p, A)$ - элемент пространства $\mathbb{P}^{m-k} \times \mathbf{S}$, в котором $\mathbb{P}^{m-k}$ идентифицируется c $P^{*}$. Заметим, что ранг $\operatorname{rk} A(p)$ не зависит от выбора координат переменной $p$ : если координаты $\left(a_{k}, \ldots, a_{m}\right)$ заменить на $\left(\lambda a_{k}, \ldots, \lambda a_{m}\right)$, то $A\left(a_{k}, \ldots, a_{m}\right)$ заменяется на $\lambda^{2 m-2} A\left(a_{k}, \ldots, a_{m}\right)$, так что ранг матрицы остается тем же и, следовательно, $\operatorname{rk} A(p)$ вполне определен для $p \in \mathbb{P}^{m-k}$. Введем

$$
\mathbf{X}_{e}=\{(p, A) \mid \operatorname{rk} A(p) \leqslant k-e\} \subset \mathbb{P}^{m-k} \times \mathbf{S}
$$

и положим

$$
\mathbf{X}_{e}(A)=\left\{p \in \mathbb{P}^{m-k} \mid \operatorname{rk} A(p) \leqslant k-e\right\}
$$

для $A \in \mathbf{S}$ и

$$
\mathbf{X}_{e}(p)=\{A \mid \operatorname{rk} A(p) \leqslant k-e\}
$$

для $p \in \mathbb{P}^{m-k}$.

Лемма 1. Если $е(e+1) / 2 \geqslant m-k+1$, то для порождающего элемента $A \in \mathbf{S}$ имеем $\mathbf{X}_{e}(A)=\varnothing$, m.е. для любого $p \in \mathbb{P}^{m-k}$ выполняется неравенство $\mathrm{rk} A(p) \geqslant k-e+1$.

ДокАЗАТЕЛЬСтво этой леммы очевидным образом получается из следующего утверждения.

Лемма 2. Для любого е $\geqslant 0$ существует непустое открытое подмножество Зарицкого $U \subset \mathbf{S}$ такое, что для $A \in U$ выполняется равенство

$$
\operatorname{codim}\left(\mathbf{X}_{e}(A) \subset \mathbb{P}^{m-k}\right)=\min \left\{\frac{e(e+1)}{2}, m-k+1\right\}
$$


ДоказАТЕЛЬСтво. Имеем

$$
\mathbf{X}_{e}(A)=\mathbf{X}_{e} \cap\left(\mathbb{P}^{m-k} \times\{A\}\right) .
$$

Пространства $\mathbb{P}^{m-k} \times\{A\}$ являются как раз слоями проектирования $\mathbb{P}^{m-k} \times \mathbf{S} \rightarrow \mathbf{S}$. Можно считать, что ограничивающее проектирование $\mathbf{X}_{e} \rightarrow \mathbf{S}$ сюръективно, поскольку если оно не сюръективно, то существует $A \in \mathbf{S}$ такое, что $\mathbf{X}_{e}(A)=\phi$, т.е. для всех $p \in \mathbb{P}^{m-k}$ выполняется неравенство $\mathrm{rk} A(p) \geqslant k-e+1$, и больше ничего доказывать не нужно. Поэтому дальнейшее доказательство этой леммы очевидным образом получается из следующего утверждения.

ЛЕмма 3. Виполняется следующее равенство:

$$
\operatorname{codim}\left(\mathbf{X}_{e} \subset \mathbb{P}^{m-k} \times \mathbf{S}\right)=\frac{e(e+1)}{2} .
$$

ДоказАтельство. Рассмотрим замкнутое алгебраическое множество $\mathbf{X}_{e}(p)=\mathbf{X}_{e} \cap$ $(\{p\} \times \mathbf{S})$. Таким образом, имеем $\operatorname{dim} \mathbf{X}_{e}=\operatorname{dim} \mathbf{X}_{e}(p)+(m-k)$, и завершение доказательства получается из следующего утверждения.

Лемма 4. Виполняется следующее равенство:

$$
\operatorname{codim}\left(\mathbf{X}_{e}(p) \subset \mathbf{S}\right)=\frac{e(e+1)}{2} .
$$

ДокАЗАтеЛЬСтво. Пусть

$$
S_{e}=\{M \in S \mid \operatorname{rk} M \leqslant k-e\} .
$$

Хорошо известно, что $\operatorname{codim}\left(S_{e} \subset S\right)=e(e+1) / 2$. Для $p=\left(a_{k}: \cdots: a_{m}\right) \in \mathbb{P}^{m-k}$ введем обозначение $\bar{p}=\left(a_{k}, \ldots, a_{m}\right) \in \mathbb{C}^{m-k+1}$ и рассмотрим отображение $\{\bar{p}\} \times \mathbf{S} \rightarrow S$, которое определяется вычислением матрицы $A \in \mathbf{S}$ в $\bar{p}$. Ясно, что это отображение сюръективно, и все слои имеют одинаковую размерность и гладкие: координаты выбираются так, чтобы $p=(1: 0: \cdots: 0)$ при координатах $x_{k}, \ldots, x_{m}$. Тогда для любого $M \in S$ прообразом будет $\{\bar{p}\} \times\left(M x_{k}^{2 m-2}+N\right)$, где $N \subset \mathbf{S}$ - подпространство матриц, элементы которых не содержат мономов $x_{k}^{2 m-2}$, а размер слоя равен

$$
\Delta=\frac{k(k+1)}{2}\left(\left(\begin{array}{c}
3 m-k-2 \\
2 m-2
\end{array}\right)-1\right) .
$$

Теперь из сказанного ранее следует, что $\mathbf{X}_{e}(\bar{p})=\mathbf{X}_{e}(p)$. Рассмотрим отображение ограничения $\mathbf{X}_{e}(\bar{p}) \rightarrow S_{e}$. Тогда, так как это отображение сюръективно, получим $\operatorname{dim} \mathbf{X}_{e}(\bar{p})=\operatorname{dim} S_{e}+\Delta$. что и требовалось доказать в данной лемме.

Напомним, что $\varphi_{E^{+}}: E^{+} \rightarrow P^{*} \cong P$ - расслоение на квадрики. Теперь элементы пространства квадратичных форм, т.е.

$$
x_{m+1}^{2}=\sum_{i, j=0}^{k-1} x_{i} x_{j} P_{i j}\left(x_{k}, \ldots, x_{m}\right)
$$

относительно $x_{0}, \ldots, x_{k-1}, x_{m+1}$ с переменными коэффициентами $P_{i j}\left(x_{*}\right)$, имеют соответствующие симметричные $(k+1) \times(k+1)$ матрицы, которые очевидным образом находятся во взаимно однозначном соответствии с элементами пространства $\mathbf{S}$. Следовательно, при заданном выборе $P_{i j}$, т.е. при заданном выборе $W$, мы получаем соответствующую матрицу $A \in \mathbf{S}$. Следовательно, подпространство в $P^{*}$, над которым квадратичные формы имеют ранг $\leqslant k+1-e$ можно идентифицировать с пространством $\mathbf{X}_{e}(A)$, и из лемм 1 и 2 следует, что это пространство либо пусто, либо имеет коразмерность $e(e+1) / 2$. В п. 1 мы предположили, что $m \leqslant k(k-3) / 2+2$. Из леммы 1 следует, что ранг любого слоя в $\varphi_{E+}$ превосходит 4 , так как $\mathbf{X}_{k-3}(A)=\varnothing$. 
5. Обращение присоединения. Теперь можно закончить доказательство нашей теоремы. Предположим, что ограниченная пара $\left(V_{\Pi}, \Sigma_{\Pi} / n\right)$ содержит точку $o \in B \cap V_{\Pi}$, которая является изолированным центром некоторой неканонической сингулярности, $\varphi: V_{\Pi}^{+} \rightarrow V_{\Pi}-$ раздутие точки $о$ и $E^{+}-$исключительный дивизор $\varphi^{-1}(o)$, который рассматривается как квадратичная гиперповерхность в $\mathbb{P}^{l}$, где $\operatorname{codim} B=l$. Далее, пусть $D^{+}$- строгое преобразование $D \in \Sigma_{\Pi}$ на $V_{\Pi}^{+}$. Существует $\mu \in \mathbb{Z}_{+}$такое, что

$$
D^{+} \sim \varphi^{*} D-\mu E^{+} \text {. }
$$

Рассмотрим пару $\left(V_{\Pi}, \Sigma_{\Pi} / n\right)$. Далее, рассуждаем как в работе [7] (см. также раздел 1 в [8]). Возьмем порождающую тройную плоскость $\Lambda \subset$ П, содержащую точку o. Положим $V_{\Lambda}=\pi^{-1}(\Lambda)$. Пусть $\Sigma_{\Lambda}-$ сужение $\Sigma$ на $V_{\Lambda}$. В силу обратного преобразования присоединения (см. [9], [10]) пара $V_{\Lambda}, \Sigma_{\Lambda} / n$ не является логарифмически канонической. Однако точка $o \in V_{\Lambda}$ - это невырожденная трехкратная квадратичная особенность. Рассуждая как в предложении 1.3 в работе [7] (или как в [11] в доказательстве предложения 3.10), приходим к выводу, что $\mu>n$.

Следовательно, mult ${ }_{o} D>2 n$ в силу (2) и того, что $E^{+}$квадратично. Однако степень $D$ равна

$$
\operatorname{deg} D=\left(D \cdot \pi^{*} H_{\Pi}^{l-1}\right)=2 n,
$$

где $H_{\Pi}-$ класс гиперповерхности в П, так как $V_{\Pi}-$ двойное накрытие и $D \sim n \pi^{*} H_{\Pi}$. Следовательно, получим mult $D>\operatorname{deg} D$, что невозможно. Это противоречие доказывает теорему.

Автор выражает глубокую благодарность своему научному руководителю Александру Пухликову за постановку задачи и многочисленные полезные обсуждения.

\section{СПИСОК ЦИТИРОВАННОЙ ЛИТЕРАТУРЫ}

[1] В. А. Исковских, Ю. И. Манин, Матем. сб., 86:1 (1971), 140-166. [2] А. В. Пухликов, УМН, 62:5 (2007), 15-106. [3] А. В. Пухликов, Изв. АН СССР. Сер. матем., 52:1 (1988), 229-239. [4] А. В. Пухликов, Алгебрачческая геометрия - 2, Итоги науки и техн. Сер. Соврем. мат. и ее прил. Темат. обз., 24, ВИНИТИ, М., 2001, 177-196. [5] И. А. Чельцов, Матем. сб., 199:2 (2008), 131-148. [6] И. А. Чельцов, Матем. сб., 195:10 (2004), 109-156. [7] A. Pukhlikov, Birational Geometry of Singular Fano Varieties, arXiv: math.AG/0807.3886. [8] A. Pukhlikov, Birational Geometry of Fano Double Spaces of Index Two, arXiv: math.AG/0812.3863. [9] В. В. Шокуров, Изв. РАН. Сер. матем., 56:1 (1992), 105-203. [10] Flips and Abundance for Algebraic Threefolds (Salt Lake City, 1991), Astérisque, 211, ed. J. Kollár, Soc. Math. France, Paris, 1992. [11] A. Corti, Explicit Birational Geometry of 3-Folds, London Math. Soc. Lecture Note Ser., 281, Cambridge Univ. Press, Cambridge, 2000, 259-312.

Р. Дж. Муллани

University of Liverpool, Великобритания

E-mail: r.j.mullany@liv.ac.uk
Поступило 30.06.2009 